\title{
The Notch locus and the genetic circuitry involved in early Drosophila neurogenesis
}

\author{
Tian Xu, Ilaria Rebay, Robert J. Fleming, T. Nelson Scottgale, and Spyros Artavanis-Tsakonas \\ Department of Biology, Yale University, New Haven, Connecticut 06511 USA
}

\begin{abstract}
The genetic and molecular analysis of the Notch locus, which codes for a transmembrane protein sharing homology with the mammalian epidermal growth factor, suggests that the Notch protein is involved in a cell interaction mechanism essential for the differentiation of the embryonic nervous system of Drosophila. Taking advantage of the negative complementation between two Notch mutations that affect the extracellular domain of the protein, we have tried to dissect the genetic circuitry in which Notch is integrated by searching for genes whose products may interact with the Notch protein. This genetic screen has led to the identification of a surprisingly restricted set of interacting loci, including Delta and mastermind. Like Notch, both of these genes belong to a group of loci, the neurogenic loci, which have been previously identified by virtue of their similar mutant phenotype affecting early neurogenesis. We extend these studies by systematically exploring interactions between specific mutations in the Notch molecule and the other neurogenic genes. Furthermore, we show that the molecular lesions of two Notch alleles (nd and $\left.n d^{2}\right)$, which interact dramatically with mastermind mutations, as well as with a mutation affecting the transducin homologous product of the neurogenic locus Enhancer of split, involve changes in the intracellular domain of the protein.
\end{abstract}

[Key Words: Notch, Drosophila; neurogenic genes; negative complementation; genetic interactions]

Received November 11, 1989; revised version accepted December 20, 1989.

In our efforts to elucidate the molecular mechanisms underlying the decision of an embryonic ectodermal cell to follow a neural versus an epidermal pathway, we have been studying a group of loci, known as the neurogenic loci, which affect this developmental process (Artavanis-Tsakonas 1988). A fundamental issue in the molecular analysis of any such process concerns the complexity of the genetic circuitry governing it. How many of the neurogenic loci revealed by mutational studies to affect the differentiation of the early ectoderm are functionally related? What other genetic elements may interact with a given neurogenic locus? In this paper we describe an approach that has allowed us to address these questions and, hence, the complexity and nature of the genetic network involved in the initial steps of neurogenesis.

The central nervous system in Drosophila is derived from a set of embryonic precursor cells, the neuroblasts, which segregate from an area of the ventral ectodermal cell layer termed the neurogenic region (Poulson 1950; Hartenstein and Campos-Ortega 1984). Within the neurogenic region, an ectodermal cell can follow either a neural or an epidermal developmental pathway. Exten-

This paper is dedicated in memory of Dr. Donald F. Poulson (1910-1989) who pioneered Drosophila developmental genetics. sive genetic screens led to the identification of six unlinked, zygotically active loci that affect this dichotomy (Lehmann et al. 1983; Jürgens et al. 1984; Nüsslein-Volhard et al. 1984; Wieschaus et al. 1984). Null mutations in any one of these loci cause embryonic lethality, and the embryos display a strikingly similar phenotype: a hypertrophy of the nervous system at the expense of epidermal structures. This phenotype suggests that cells destined to become epidermal precursors change fate and become neuroblasts (Poulson 1937; Lehmann et al. 1983). These six zygotic neurogenic loci are Notch $(N)$, Delta (DI), Enhancer of split [E(sp1)], mastermind (mam), big brain (bib), and neuralized (neu).

The genetic and molecular analysis of $N$, the best characterized among the neurogenic loci so far, suggests that the $N$ protein is involved in a cell interaction mechanism that mediates the differentiation of the embryonic nervous system (Wharton et al. 1985a). Sequence data revealed that it encodes a 2703 -amino-acid transmembrane protein whose extracellular domain shows homology to the mammalian epidermal growth factor [EGF (Wharton et al. 1985a; Kidd et al. 1986)]. The structure and the location of the $N$ protein are consistent with a role in mediating information exchange between neighboring cells (Johansen et al. 1989; Kidd et al. 1989), and embryological studies both in the grasshopper and 
in Drosophila support such a cell interaction mechanism during early ectodermal development (Doe and Goodman 1985; Technau and Campos-Ortega 1986). The accumulated genetic and molecular evidence demonstrates that $N$ is not exclusively involved in embryonic development but is also required postembryonically. Many $N$ mutations affect various adult structures, and the protein is expressed in various tissues during development (Welshons 1965; Hartley et al. 1987; Johansen et al. 1989; Kidd et al. 1989; Markopoulou and Artavanis-Tsakonas 1989). The correct differentiation of certain adult structures (e.g., retina and bristles) has been shown to require both $N$ function and cell-cell interactions (Shellenbarger and Mohler 1978; Tomlinson and Ready 1987; Cagan and Ready 1989; also see review by Held and Bryant 1984). $N$ seems to play a more general role in development than would have been expected from a gene merely involved in the segregation of neural from epidermal lineages in the embryo; hence, the postulated cell interaction mechanism in which it is involved may function throughout ontogeny (Hartley et al. 1987; Artavanis-Tsakonas 1988; Markopoulou and Artavanis-Tsakonas 1989). The concept of regulation of early neurogenesis, as well as postembryonic development by a cell interaction mechanism implies several interacting components. One possible way of identifying these components is to search for genetic units interacting with $N$.

Genetic analyses have led to the identification of three mutant classes in the $N$ locus. The first class includes the neurogenic $N$ mutations, apparent "null" alleles, which behave as recessive lethals and in heterozygous condition display a haploinsufficient phenotype affecting mainly wing and bristle development (Welshons 1965, 1971). The second class includes the recessive visible mutations [e.g., the facet (fa), split (spl), and notchoid (nd) alleles], which are associated with eye, bristle, and/or wing abnormalities. The third class of $N$ mutations consists of the dominant Abruptex $(A x)$ mutations, which exhibit a phenotype of gapped wing veins and bristle abnormalities. The $A x$ mutations display intriguing genetic behavior. There are two types of $A x$ alleles: recessive viables and recessive lethals. The viable mutants subdivide into two groups based on their interactions with $N$ alleles. In heteroallelic combinations, one group of $A x$ mutants suppresses the $N$ wing nicking phenotype and the other enhances it. Among these viable $A x$ alleles, transheterozygotes within each group are viable, whereas those between groups are lethal, and transheterozygotes of any viable $A x$ allele with any lethal $A x$ allele are also lethal, a phenomenon known as negative complementation (Welshons 1971; Foster 1975; Portin 1975). The molecular analysis of these $A x$ alleles revealed that they are missense mutations in the extracellular EGF homologous region of the $N$ protein (Hartley et al. 1987; Kelley et al. 1987). Because transheterozygotes of any viable $A x$ allele over $N$ deficiencies are viable, the lethality of negative complementation between $A x$ alleles cannot be explained as the result of the reduction of the amount of the $N$ protein but, rather, as the result of interactions between two types of mutated $N$ proteins.
The negative complementation between $A x$ alleles gives us a powerful tool to systematically dissect the genetic circuitry in which $N$ is integrated. Supposing that a particular gene product interacts with $N$ at the protein level, it is conceivable that mutations in that gene may interfere with negatively complementing $A x$ alleles. We therefore sought to identify suppressors of the lethality conferred to the animal by the negative complementation between two viable $A x$ alleles: the enhancer type $A x^{E 2}$ and the suppressor type $A x^{9 B 2}$. These two mutants are associated with missense changes in the extracellular EGF-like region of the $N$ protein [single missense changes: histidine to tyrosine and aspartic acid to valine, respectively (Hartley et al. 1987; Kelley et al. 1987)]. This screen should reveal both second site mutations within $N$ and mutations in unlinked loci whose products may interact with the $N$ protein. In this paper we report the results of such a screen, which led to the identification of a surprisingly restricted set of loci. We extended these studies by systematically exploring allele-specific interactions between $N$ and the other zygotic neurogenic genes. Finally, we determined the molecular lesions associated with $n d$ and $n d^{2}$ in an attempt to gain insight into the molecular nature of their dramatic interaction with mutations in the mam and $E(s p l)$ loci.

\section{Results}

The screen for suppressors of negative complementation between $\mathrm{Ax}$ mutations of the $\mathrm{N}$ locus

The screen carried out to identify suppressors of the lethality of negative complementation between $A x^{E 2}$ and $A x^{9 B 2}$ is summarized in Figure 1. Homozygous $A x^{E 2} s n^{3}$ virgins were mated to ethylnitrosourea (ENU)-treated $y$ $W A x^{9 B 2}$ males. In the next generation, whereas the $A x^{E 2}$ $s n^{3}$ males develop into adults, the $A x^{E 2} s n^{3 / y} w A x^{9 B 2}$ transheterozygous females die as a result of negative complementation unless a dominant mutation capable of rescuing such lethality has been induced in the genome. In this screen, 36 fertile $A x^{E 2} s n^{3 / y}$ w $A x^{9 B 2}$ transheterozygous females were recovered among 12,946 sibling $A x^{E 2} s n^{3}$ males.

Because either one of the two $A x$ alleles is viable over a null $N$ allele, it is not surprising that the subsequent genetic mapping of the isolated suppressors demonstrated that the majority (23) were X-linked. Additional genetic mapping revealed that 21 of them were lethal $N$ alleles, whereas the remaining two were viable mutations, the map positions of which remain to be fully characterized.

Mapping of the autosomal suppressors showed that they fell into two lethal complementation groups, one on the second chromosome and the other on the third. There were four mutations in the first group and nine in the second. Phenotypic analysis raised the possibility that the two groups might be allelic to the neurogenic loci mam and $D l$, respectively. Supressors on the third chromosome were genetically mapped to the interval in which $D 1$ is located. Indeed all nine suppressor muta- 


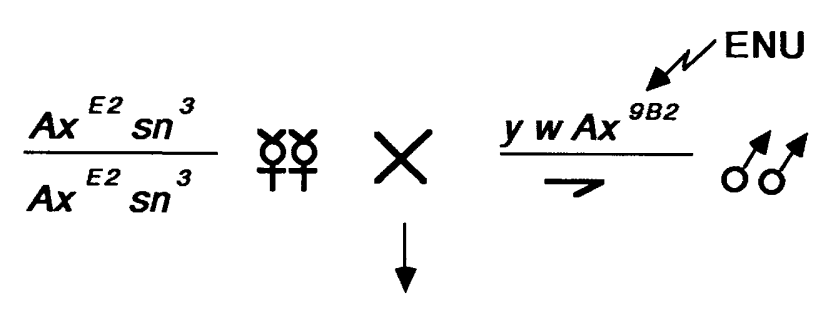

36 Viable, Fertile

$\frac{y w A x^{9 B 2}}{A x^{E 2} s n^{3}}$

12,946

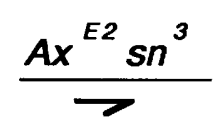

Figure 1. Suppressor screen. The genetic scheme for selecting suppressors of the negative complementation between the $A x^{E 2}$ and $A x^{9 B 2}$ alleles of the $N$ locus. Whereas the majority of the heterozygous Fl females died, 36 fertile, transheterozygous $A x$ females were collected from among 12,946 $A x^{E 2} s n^{3}$ sibling males. Individual lines were established. (For mapping details, see Materials and Methods.)

tions on the third chromosome failed to complement $D 1^{B X 6}$ and $D 1^{9 P 39}$, two distinct loss-of-function $D l$ alleles (Alton et al. 1989; ref. in Lindsley and Zimm 1985). Similarly, the four mutations on the second chromosome failed to complement mam ${ }^{I L 115}$ (Nüsslein-Volhard et al. 1984). We conclude that the autosomal suppressors that we have isolated are mam and $D l$ alleles. (For details of the genetic crosses and allele names, see Materials and methods|. It should be noted that the animals rescued by $D l$ and mam mutations are not wild type. They display an $A x$ phenotype that is more severe than either $A x^{E 2}$ or $A x^{9 B 2}$ homozygotes (data not shown).

\section{$\mathrm{Dl}$ and mam alleles suppress all lethal $\mathrm{Ax}$ combinations}

Although the preliminary analysis of the $\mathrm{Dl}$ and mam alleles isolated from the screen indicated that some of them were hypomorphic alleles (M.A.T. Muskavitch, pers. comm.|, we found that the previously existing neurogenic $D I$ alleles $\left[D f(3 R) D 1^{B \times 6}, D 1^{9 P 39}\right]$ and mam allele $\left(\mathrm{mam}^{I L 115}\right)$ (Nüsslein-Volhard et al. 1984) were also able to rescue the lethality of $A x^{E 2} / A x^{9 B 2}$ animals. $D f(3 R) D l^{B X 6}$ is a cytologically visible deficiency of $D l$, and $D 1^{9 P 39}$ also appears to be amorphic (Lehman et al. 1983). The severity of the mam $^{I L 115}$ phenotype also suggests that it, too, is an amorphic mutation. Therefore, this result suggests that the simple reduction to one copy of either $D l$ or mam is capable of suppressing the lethality associated with the negative complementation (Fig. 2A).

These observations were extended by examining further aspects of the $A x, D I$, and mam interactions. We were particularly interested in whether the suppression of the lethality was specific for these two alleles (i.e., $A x^{E 2}$ and $\left.A x^{9 B 2}\right)$. The effects of $D I$ and mam on the nega-

tive complementation between combinations of six different $A x$ alleles were examined $\left(A x^{9 B 2}\right.$, the viable, suppressor type; $A x^{E 2}, A x^{71 d}$, and $A x^{16172}$, the viable enhancer types; and $A x^{75 C 24}$ and $A x^{59 d}$, the two recessive lethal alleles). The results summarized in Figure 2A indicate that mam and $D I$ mutations were generally capable of suppressing the lethality conferred by all the negatively complementing $A x$ heteroallelic combinations tested. Thus, the rescue of negative complementation is not specific to the $A X^{E 2} / A x^{9 B 2}$ combination. However, the effect that the $D l$ and mam mutants had on the phenotypes of individual $A x$ alleles in hemizygous males was not the same: Whereas $D l$ mutations generally suppressed the phenotypes of the viable $A x$ alleles, but not the lethality of lethal $A x$ alleles (Fig. 2B; Fig. $4 C$ ), mam mutations did not seem to have any effect on the individual $A x$ phenotypes (Fig. 2B).

Although neu, bib, and $E(s p l)$ mutations were not recovered by the screen, we directly examined their effects on the negative complementation between $A x$ alleles. The mutations included $b i b^{I D 05}$ from the bib locus and $n e u^{I F G 5}$ from the neu locus. Three different types of mutations affecting the $E$ (spl) locus were also tested: (1) the two deficiencies $E(s p l)^{R 1}$ and $1(\text { gro })^{X 1}$, which involve several chromosome bands and thus affect simultaneously many transcription units in the $E(s p l)$ region (Knust et al. 1987; Preiss et. al. 1988); (2) the lethal point mutation $E(s p l)^{E 73}$ which, as previously reported, affects the $\beta$ transducin homologous transcript of the region (Hartley et al. 1988; Preiss et al. 1988); and (3) the dominant $E(s p l)^{D}$ allele which is known to interact with the $N$ allele split (Welshons 1956). As might have been predicted from the results of the screen, none of these mutations was capable of suppressing the negative complementation between $A x^{E 2}$ and $A x^{9 B 2}$. In addition the $P\left[\mathrm{ry}^{+} ; E 8\right]$ transposon carrying DNA, which was shown to be capable of rescuing the lethal point mutations in the $E(s p l)$ region (Preiss et al. 1988), was also unable to rescue the $A x^{E 2} / A x^{9 B 2}$ lethality. These data are summarized in Figure 2A. The effects of these mutations on the phenotypes of individual $A x^{E 2}$ or $A x^{9 B 2}$ alleles were also examined. As indicated in Figure $2 B$, the only mutations that seemed to interact with the individual $A x$ phenotypes are the $E(s p l)$ deficiencies and the $E$ (spl) lethal point mutations. It should be noted that the effect was not the same: the deficiencies caused phenotypic suppression (Fig. 4D), whereas the point mutations $\left[E(s p l)^{E 73}\right.$, $E(s p l)^{E 48}$, and $\left.E(s p l)^{E 107}\right]$ caused enhancement (Fig. 4H).

In summary, this analysis shows that the reduction of the wild-type copy number of $D l$ (and $\mathrm{mam}$ ) was capable of interfering with the mechanism underlying negative complementation in a manner that was not restricted to specific $A x$ combinations. Because there was no correlation between suppressing the individual $A x$ phenotype and rescuing the lethality between them, the simplest hypothesis to explain these results is that the rescue of the negative complementation is not due to the complete suppression of one of the $A x$ alleles but, rather, is due to an interference with interactions between the mutated $N$ alleles. 


\begin{tabular}{|c|c|c|c|c|c|c|c|c|c|c|c|}
\hline & $\begin{array}{l}\text { A. } \\
\frac{A x^{E 2}}{A x^{9 B 2}}\end{array}$ & $\frac{A x^{71 d}}{A x^{9 B 2}}$ & $\frac{A x^{16172}}{A x^{982}}$ & $\frac{A x^{E 2}}{A x^{75 C 24}}$ & $\frac{A x^{E 2}}{A x^{59 d}}$ & $\begin{array}{l}\text { B. } \\
\frac{A x^{E 2}}{\longrightarrow}\end{array}$ & $\frac{A x^{71 d}}{\longrightarrow}$ & $\frac{A x^{16172}}{\longrightarrow}$ & $\frac{A x^{9 B 2}}{-}$ & $\frac{A x^{75 c 24}}{\longrightarrow}$ & $\frac{A x^{59 d}}{\longrightarrow}$ \\
\hline $1+$ & $\mathrm{R}$ & R & $R$ & $R$ & $R$ & $s$ & $s$ & $n c$ & $S(w)$ & nc & nc \\
\hline $1+$ & R & $\mathbf{R}$ & R & & & $n c$ & $n c$ & $n c$ & nc & & \\
\hline$E(s p l) \stackrel{\text { deficiencies }}{1+}$ & $\mathrm{nR}$ & $\mathrm{nR}$ & $\mathrm{nR}$ & & & $S$ & & & $S(w)$ & & \\
\hline$E(s p l)^{\text {points }} /+$ & $\mathrm{nR}$ & & & & & $E$ & & & $E$ & & \\
\hline$E(s p l)^{D} /+$ & $\mathrm{nR}$ & & & & & $n c$ & & & $n c$ & & \\
\hline$P[r y+; E 8]{ }^{*}$ & $\mathrm{nR}$ & & & & & $n c$ & & & $n c$ & & \\
\hline bib & $\mathrm{nR}$ & $\mathrm{nR}$ & $\mathrm{nR}$ & & & $n c$ & & & $n c$ & & \\
\hline neu & $\mathrm{nR}$ & $n R$ & $n R$ & & & nc & & & $n c$ & & \\
\hline
\end{tabular}

Figure 2. The negative complementation of the $A x$ alleles and its relationship to neurogenic mutations. $(A)$ The effects of mutations at different neurogenic loci on various negatively complementing $A x$ alleles are summarized. The top row indicates the genetic constitution of the $\mathrm{X}$ chromosome, and the left-most vertical column indicates the autosomal genetic constitution. (R) Rescued (viable); $(\mathrm{nR})$ not rescued (lethal). Empty spaces indicate untested combinations $(*) . P\left[r y^{+} ; E 8\right]$ is a transformed line that contains DNA

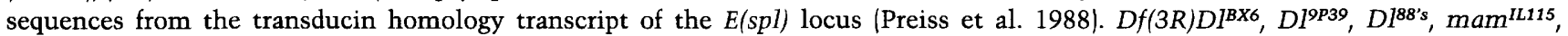

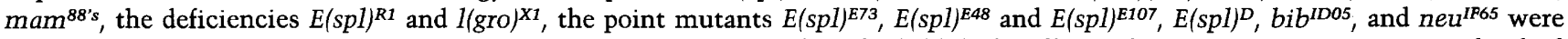
used in the tests. (For details of strains and crosses, see Materials and methods.) (B) The effects of neurogenic mutations on individual $A x$ phenotypes is presented as not changed (nc), weakly suppressed [S(w)], suppressed (S) or enhanced (E). Note that nc for the $A x^{75 C 24}$ and $A x^{59 d}$ lethal alleles indicates nonviability (i.e., not rescued). The organization is as in $A$.

\section{Interactions between the $\mathrm{N}$ mutant classes and the zygotic neurogenic loci}

Previous intragenic mapping of the various existing $N$ alleles in conjunction with the molecular analysis of the gene has permitted an association of specific mutations with domains of the $N$ molecule (for review, see Artavanis-Tsakonas 1988). The analysis described so far revealed interactions between some of the neurogenic loci and a class of $N$ alleles that affect the extracellular EGFlike domain of the molecule. If some or all of the zygotic neurogenic loci actually code for interacting products, it is conceivable that the interaction profiles of mutants affecting different parts of the $N$ molecule will be different. We have thus examined the phenotypic interactions between specific $N$ alleles and other neurogenic mutations in a manner analogous to that described above for the individual $A x$ mutations.

Figure 3 summarizes the results we obtained when animals were tested in a double-mutant condition, where one copy of the mutant neurogenic gene was combined with a particular $N$ mutation. The top of Figure 3 is a schematic representation of the $N$ gene, with the approximate position, molecular lesion, and phenotypic description of each $N$ mutation examined. In summary, none of the $N$ alleles interacts with either bib or neu mutations. In contrast, we observe a complex, allele-specific pattern of interactions between several $N$ mutations and the remaining neurogenic loci. Several aspects of the observed interactions warrant comment.

A rather dramatic interaction was observed between the two $N$ alleles $n d$ and $n d^{2}$ and the $E$ (spl) point mutation $E(s p l)^{E 73}$. These two $N$ alleles map farthest to the $3^{\prime}$ end of the locus (Welshons 1971; see also Fig. 5) and have distinct, but very similar, phenotypes. Both of these mutations in a homozygous or hemizygous form display wing notching and have thickened veins, as indicated in Figure $4 \mathrm{E}$. In addition, $n d^{2}$ displays wing vein gaps (data not shown). Animals heterozygous for $E(s p l)^{E 73}$ are phenotypically wild type. However, nd animals that were also heterozygous for $E(s p l)^{E 73}$ (nd/Y; $E(s p l)^{673} /+$ ) displayed a severe $100 \%$ penetrant wing phenotype shown in Figure 4F. The same synergistic effects were observed with $n d^{2}$ animals (data not shown), demonstrating a dramatic interaction between what seems to be a point mutation in the $E(s p l)$ transducin homologous transcript and $N$.

A noteworthy interaction was also observed between $n d^{2}$ and another mutation associated with the $E(s p l)$ locus, the dominant $E$ (spl) allele. This mutation maps to 


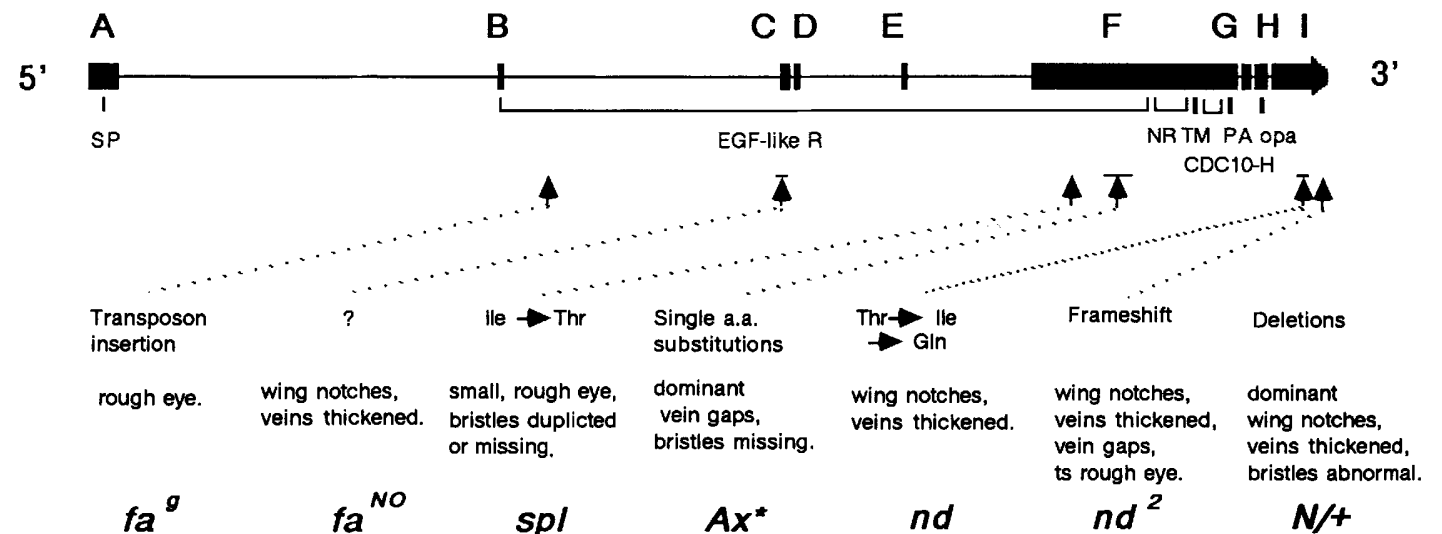

\begin{tabular}{|c|c|c|c|c|c|c|c|}
\hline & $f a^{g}$ & $\mathrm{fa}^{\mathrm{NO}}$ & $s p l$ & $A x^{*}$ & $n d$ & $\begin{array}{l}\text { ts rough eye. } \\
n^{2} d^{2}\end{array}$ & $\begin{array}{l}\text { bristles abnormal } \\
\mathrm{N} / \mathrm{t}\end{array}$ \\
\hline$D I$ & nc & $S(w)$ & nc & $S$ & $S(w)$ & E : eye & Viable,S(w) \\
\hline $\operatorname{mam}$ & nc & $E(w)$ & $S(w)$ & nc & $E(s)$ & E : wing & Viable, nc \\
\hline$E(s p l)^{\text {deficiencies }} /+$ & nc & nc & nc & S & nc & nc & $\begin{array}{l}\text { Viable, n c } \\
\text { or lethal }\end{array}$ \\
\hline$E(s p l)^{E 73} /+$ & nc & $n c$ & $\begin{array}{c}A x^{\dagger} \\
n c: s p l\end{array}$ & E : bristles & $E(s)$ & E : wing & Viable, E \\
\hline$E(s p l)^{D} /+$ & $n c$ & nc & $E(s): e^{t+\infty}$ & nc & nc & E : eye & Viable, nc \\
\hline $1+$ & nc & nc & nc & nc & nc & nc & Viable, n c \\
\hline neu & nc & nc & nc & nc & nc & nc & Viable, nc \\
\hline
\end{tabular}

Figure 3. Interactions between neurogenic mutations with specific $N$ alleles. The genomic organization of the $40-\mathrm{kb}$-long $N$ locus is schematically shown at top: The nine exons (A to I) give rise to a 10.1-kb mRNA and are indicated by solid bars connected by lines representing the intronic regions. The various key domains of the corresponding $N$ protein are denoted below by lines and brackets: (SP) Signal peptide; (EGF-like R) EGF-like repeats; (NR) cysteine-rich repeats present in $N$; (TM) transmembrane domain; (CDC10-H) repeats of yeast CDC10 homologous sequence (Breeden and Nasmyth 1987); (PA) nucleotide phosphate binding sequence homology; (opa) opa repeat (Wharton et al 1985b). (For more details, see Wharton et al. 1985a.) The approximate positions (arrows) of the molecular lesions and the phenotypes of different $N$ alleles are also depicted. The $\mathrm{X}$ chromosome constitution of the animals is given in the top row of the table, and the relevant autosomal constitution is presented in the first column. The observed $N$ allele phenotypes in the double-mutant combinations are presented as follows: (nc) Not changed; (S) suppressed or [S(w)] weakly suppressed; (E) enhanced, $[\mathrm{E} / \mathrm{w})]$ weakly enhanced, or $[\mathrm{E} / \mathrm{s})]$ strongly enhanced. Cases in which only certain aspects of the phenotype are enhanced are indicated after (E). For example, E : eye means that the eye phenotype is enhanced. (For details of strains and crosses, see Materials and methods.) (*) Generalized results from Fig. 2B. (**)The vein thickened phenotype of $N$ is weakly suppressed and the $D 1$ wing vein phenotype is suppressed. $\left(^{\star \star *}\right)$ When the $E($ spl) alleles are provided from the mothers, such double heterozygous animals are lethal; otherwise they are viable. $(t)$ The $s p l$ phenotype is nc; however, a novel $A x$-like wing phenotype is observed; see Fig. 4G. $(\dagger+)$ The rough eye phenotypes are more severe at low temperature $\left(18^{\circ} \mathrm{C}\right)$ than at room temperature $\left(25^{\circ} \mathrm{C}\right) .(@)$ These phenotypic interactions have been reported previously (Welshons 1956; Vässin et al. 1985).

Figure 4. Phenotypic interactions among mutations of $N, D l$, mam, and $E(s p l)$ loci. $(A) A x^{E 2} s n^{3} / Y$ male: Note the typical wing vein gaps at the posterior ends of the fourth and fifth longitudinal veins. $(B) w \mathrm{spl} / Y$ male: The wing morphology is phenotypically normal; the $s p l$ mutation causes small rough eyes and an abnormal bristle pattern. $(C) A x^{E 2} s n^{3 / Y}$; $D 1^{9 P 39} /+$ male: The phenotype (wing vein gaps) of the $A x^{E 2}$ mutant is almost completely suppressed by the $D l$ mutation. A weak $D 1$ wing vein phenotype is also present. (D) $A x^{E 2} s n^{3} / Y ; E(s p l)^{R 1} /+$ male: The phenotype (wing vein gaps) of the $A x^{E 2}$ mutant is almost completely suppressed by $E$ (spl) deletions. $(E) w^{a} n d / Y$ male: Shows typical distal wing notches. $(F) w^{a} n d / Y ; e^{4} E(s p l)^{E 73} t x /+$ male: A completely penetrant severe wing material loss phenotype is observed in this genetic combination. In addition, double heterozygote females, $w^{a} n d /+; e^{4} E(s p l)^{E 73} t X /+$, display a $n d$ wing phenotype similar to homozygous $n d$ animals. $(G) w s p 1 / Y ; e^{4} E(s p l){ }^{E 73} t x /+$ male: This combination exhibits an $A x-l i k e$ phenotype (a small gap at the posterior end of the fifth longitudinal vein), whereas the spl phenotype remains unchanged. This effect is

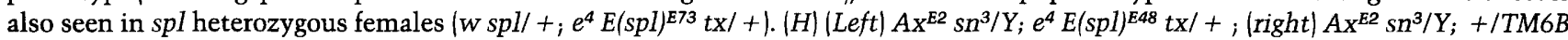
males: The left sibling displays the enhanced micro- and macrochaetae loss phenotype (indicated by arrows) when both $A x$ and $E(s p l)$ point mutations are present (cf. to control sibling at right). The analogous combination of the $E(s p l)^{E 73}$ allele with the $A x^{E 2}$ and $A x^{9 B 2}$ mutation shows a similar phenotype. Another weak point mutation, $E(s p 1)^{E 107}$, in combination with $A x^{E 2}$, causes loss of bristles on the head but not the thoracic regions. 
a transcript that is adjacent to the transducin-like transcription unit affected by the point mutations mentioned above (Knust et al. 1987; Klaembt et al. 1989). $n d^{2}$ flies have a temperature-sensitive rough eye phenotype (Shellenbarger and Mohler 1975). One copy of the domi-
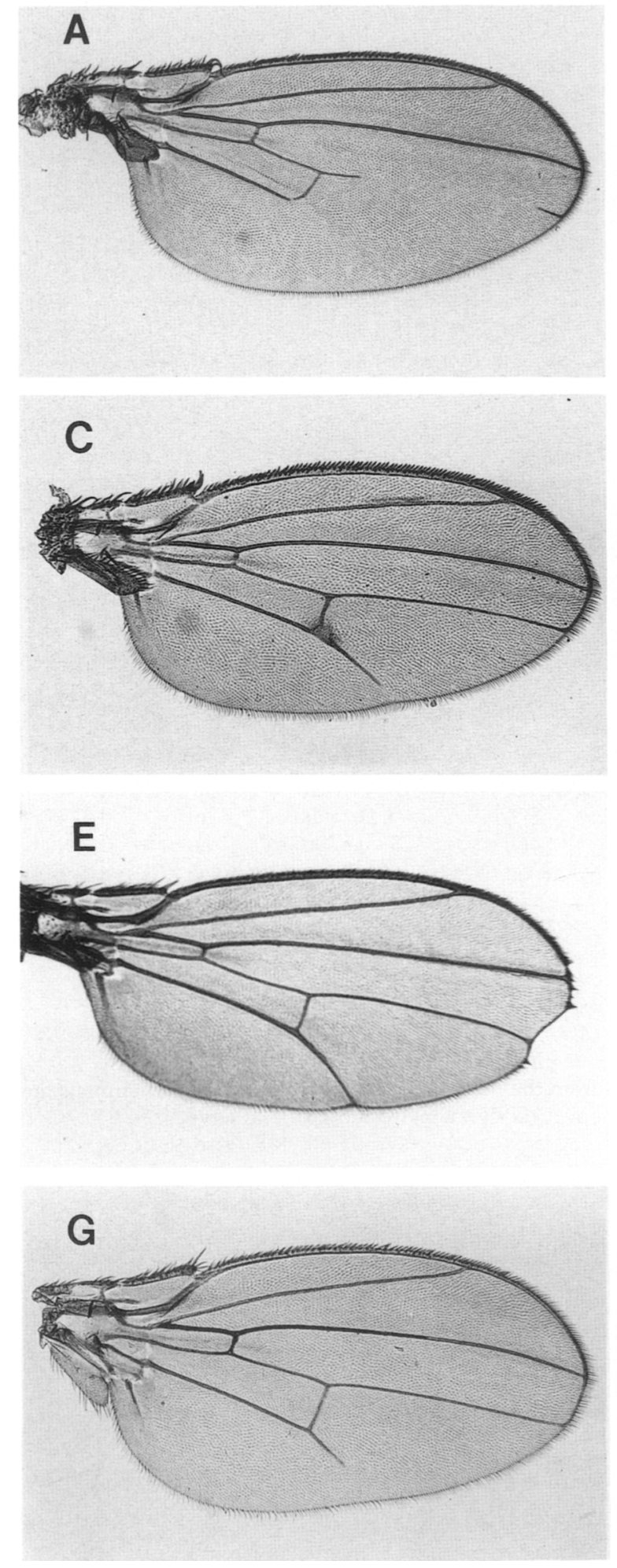

nant $E(s p l)$ allele enhances this $n d^{2}$ rough eye phenotype. Moreover, at low temperature $\left(18^{\circ} \mathrm{C}\right)$, the rough eye phenotype was affected more severely than at room temperature (data not shown).

Furthermore, the $n d$ and $n d^{2}$ alleles interact with both

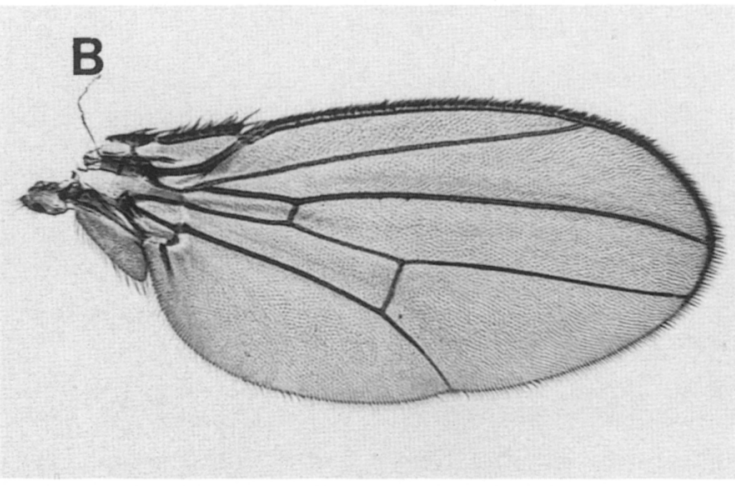

D

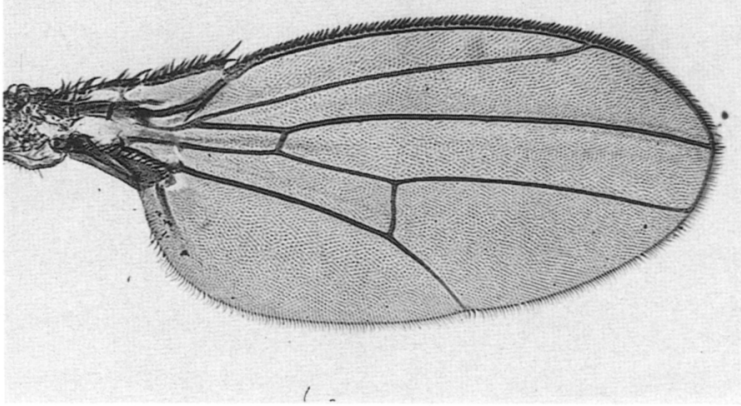

F

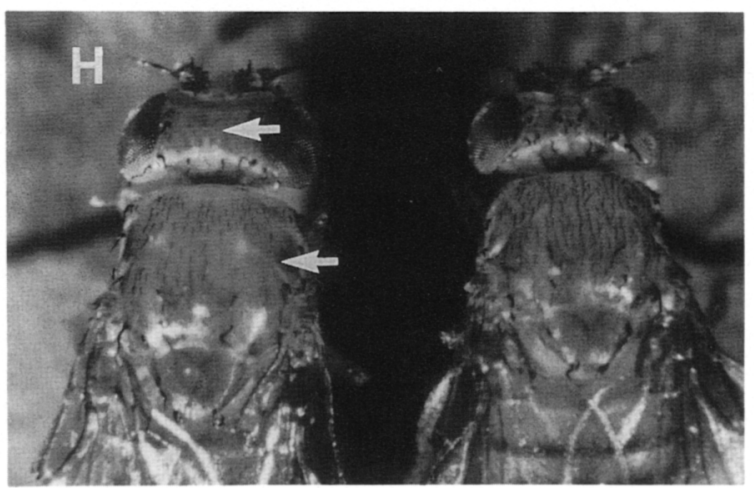

Figure 4. (See facing page for legend.) 
$D 1$ and mam. An interaction very similar to that seen between the $E$ (spl) lethal point mutation and the two nd alleles was also observed between mam and these mutations. In hemizygous $n d$ males carrying one copy of a mam mutation (nd/Y; $\operatorname{mam}^{I L 115 /}+$ ), the development of the wing was severely affected /similar to that shown in Fig. 4F). It should be pointed out that the interaction between $n d$ and $E(s p l)^{E 73}$ described above was not identical to that seen between nd and mam: The double heterozygotes of $n d$ and $E(s p l)^{E 73}\left(n d /+; E(s p l)^{E 73 /+1 \text { had a nd }}\right.$ wing phenotype like homo- or hemizygous nd flies, whereas the double heterozygotes of nd and mam (nd/ + ; $\operatorname{mam}^{I L 115 /+\mid}$ were phenotypically wild type. In contrast, the wing notching of hemizygous nd males with one copy of $D 1$ was actually suppressed, producing an almost wild-type wing (data not shown).

Unlike the enhancing effect mam mutations have on the $n d$ phenotype, an opposite effect is observed with spl. spl is a mutation affecting the extracellular domain of $N$ (Hartley et al. 1987; Kelley et al. 1987), which causes small rough eyes and duplicated bristles. The eyes of hemizygous spl animals with one copy of a mam mutation $(\mathrm{spl} / \mathrm{Y} ; \mathrm{mam} /+$ ) were less rough and larger than those carrying the $s p l$ mutation alone. At the same time, the $s p l$ bristle phenotype was also reduced. Different mam alleles had differing degrees of effects on the $s p l$ phenotype. mam $^{88-4}$ and $\operatorname{mam}^{I L 115}$ caused a clear suppression of both the eye and bristle phenotypes associated with $s p l$, whereas $\operatorname{mam}^{88-10}$ suppressed only very weakly.

These studies also revealed a phenotypic interaction between the point mutant $E(s p l)^{E 73}$ and $s p l$. Both hemizygous and heterozygous $s p l$ mutants with one copy of $E(s p l)^{E 73}\left[s p l / Y_{;} E(s p l)^{E 73} /+\right.$ or $s p l /+; E(s p l)^{E 73 /+]}$, while showing an unmodified $s p l$ phenotype, also displayed an $A x$-like phenotype, namely small gaps in the posterior end of the fifth longitudinal wing vein (Fig. 4B versus $G$ ). Finally, we noted that the rough eye phenotype of $\mathrm{fa}^{\mathrm{g}}$, a mutant associated with the insertion of a transposable element in an intronic region and, hence, thought to affect $N$ transcription (Kidd and Young 1986; Markopoulou et al. 1989|, was not modified by mutations in any one of the other zygotic neurogenic loci.

\section{Molecular lesions associated with $\mathrm{nd}$ and $\mathrm{nd}^{2}$}

We determined the molecular lesions associated with $n d$ and $n d^{2}$ to gain more insight into the nature of their interactions with mutations in the other neurogenic loci. As already mentioned, intragenic recombination analysis had shown that these two alleles map at the extreme proximal (i.e., 3') end of the locus (Fig. 5). Thus, both presumably affect the intracellular domain of the $N$ protein.

Sequence analysis of $n d^{2}$ genomic DNA revealed the deletion of $1 \mathrm{bp}$ in codon 2690 , causing a frameshift that changes the 14 carboxy-terminal amino acids into a new sequence, which is 23 amino acids in length. Similar analysis of the nd mutation, which maps distal (i.e., 5') to $n d^{2}$, uncovered two lesions: (1) a 3-bp insertion at the $3^{\prime}$ end of the polyglutamine stretch (the opa repeat; Wharton et al. 1985b) which results in the addition of an extra glutamine; and (2) a missense mutation in the codon of amino acid 2453 (Wharton et al. 1985a), resulting in a threonine to isoleucine change. Because both of these mutations in $n d$ map distally to the $n d^{2}$ lesion, any one or both could cause the mutant phenotype. Further experiments are necessary to distinguish between these possibilities. A summary of these results is depicted in Figure 5. Also indicated in Figure 5 (A; vertical lines) are the genomic regions that were sequenced in $n d$ and $n d^{2}$. In the case of $n d$, which maps genetically distal to $n d^{2}$, the sequence analysis included the genomic sequences from the transmembrane domain to the polyadenylation site. We note that the nd chromosome has an identical 3 '-untranslated sequence to the wild type, whereas $n d^{2}$ has only a single nucleotide difference in the same region. The intronic regions were also found to be very similar between the different chromosomes. Only 3-bp changes were found in the intron between exon $\mathrm{G}$ and $\mathrm{H}$ in the $n d$ chromosome and no changes were found in the $n d^{2}$ chromosome. The actual changes are indicated in the legend to Figure 5.

\section{Discussion}

Genetic analyses in Drosophila have suggested that certain basic developmental events are under the control of rather small groups of genes (Nüsslein-Volhard and Wieschaus 1980). Mutation of any member of a particular group can lead to similar phenotypes, raising the possibility that together, they define a developmental pathway. Following this rationale, the zygotic neurogenic loci, which all result in a similar hypertrophy of the embryonic CNS when mutated, could define a single developmental pathway. Although conventional mutational screens can recover mutations that produce a similar phenotype, they cannot reveal the relationships between these genes. In addition, genes involved in the same developmental process may be recalcitrant to mutational analysis because of functional redundancies. Screens involving simultaneously two or more mutations address both of these issues. In an effort to dissect the genetic circuitry in which $N$ is integrated, we have carried out such a screen, taking advantage of the negative complementation observed between $A x$ alleles. We expected this to be a general screen for genes whose products may interact with $N$, so we did not necessarily expect to identify a very limited number of loci. Because no a priori assumptions were made, it is even more surprising that null and hypomorphic alleles of $D I$ and mam were the only autosomal suppressors identified. This observation, along with the phenotypic analyses of $D 1$ and mam, indicates strongly that $N$ and these two loci are integrated in the same genetic circuitry, possibly through direct protein-protein interactions. In addition to these two neurogenic loci, the screen led to the identification of one novel, $\mathrm{X}$-linked gene that can suppress the $A x$-negative complementation. Preliminary results suggest that one of the two X-linked viable suppressors 
A.

$5^{\prime}$

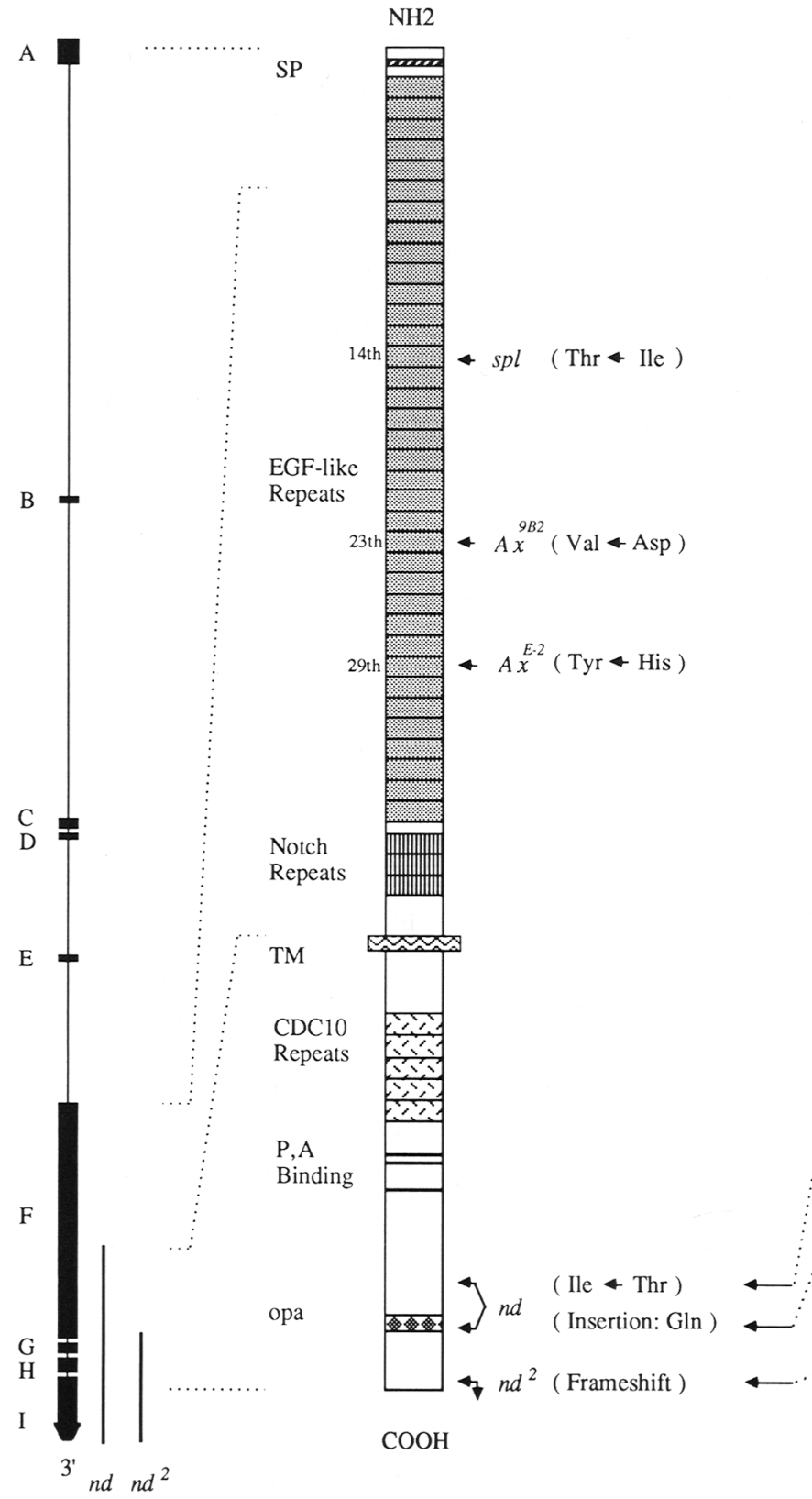

B.

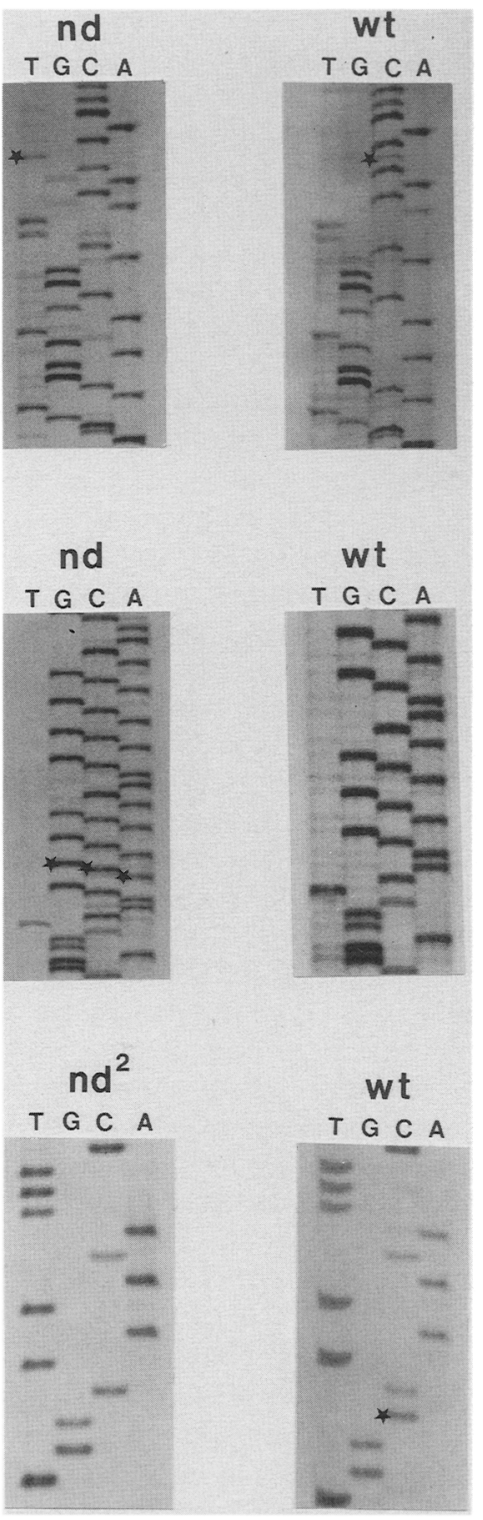

Figure 5. (A) (Left) The genomic organization of the $N$ locus; (right) the 2703 -amino-acid $N$ protein. The dotted lines between the two correlate the protein to the approximate position of the corresponding coding sequence. (The genomic organization of the $N$ locus and the different domains of the corresponding $N$ protein are labeled as in Fig. 3.) The vertical lines parallel to the genomic representation of $N$ indicate the regions of genomic DNA of the $n d$ and $n d^{2}$ chromosomes that were sequenced. Indicated to the right of the protein depiction is the position of the $n d^{2}$ mutation, a cytosine deletion that causes a frameshift at amino acid 2690 , thereby changing the 14 terminal amino acids into a new 23-amino-acid sequence (5'-Val-Ala-Ile-Arg-Leu-Thr-Arg-Val-Pro-Arg-Pro-Ser-Thr-Phe-Asp-Arg-AspLeu-Asp-Asp-Leu-Val-Cys-3'). The positions of the nd mutational changes, a cytosine to thymine transition, which causes the threonine at amino acid 2453 to change to an isoleucine, and a 3-base insertion (guanidine, cytosine, adenine) resulting in an additional glutamine insertion between amino acids 2564 and 2568 in the opa repeat, are indicated as well. One additional nucleotide change was found in the $3^{\prime}$-untranslated region of the $n d^{2}$ allele: $\mathrm{C}$ to $\mathrm{T}$ at base 9233 (Wharton et al. 1985a). It is also noted that three additional nucleotide changes between wild-type and nd chromosomes are found in the intron separating exons $G$ and $H$. Their positions within that intron and the changes are as follows: A to G (16) and T to A (49 and 64) (Kidd et al 1986; Wharton 1986). In addition, five silent nucleotide substitutions were found in the coding region of the nd chromosome: $\mathrm{A}$ to $\mathrm{G}$ at base 7233 ; $\mathrm{C}$ to $\mathrm{T}$ at bases 7914,8580 , and 8691 ; $G$ to $A$ at base 8390 (Wharton et al. 1985a). Finally for comparative purposes the positions and the amino acid changes of $s p l, A x^{9 B 2}$, and $A x^{E 2}$ mutations are also indicated. $(B)$ Autoradiographs of sequencing gels showing parts of the $n d$ chromosome sequence, the $n d^{2}$ chromosome sequence, and the corresponding wild-type (wt) chromosome sequence (Wharton et al. 1985a) indicate the nucleotides that differ between the mutant and wild-type genes. 
is allelic to the previously identified deltex locus ( $\mathrm{T}$. Xu and S. Artavanis-Tsakonas, unpubl.).

Because the lethal phenotype of an animal heterozygous for a negatively interacting $A x$ allele pair can be suppressed by the complete inactivation of one of the $A x$ alleles, the question arises whether the underlying cause for the observed suppression is actually the inactivation of one of the $A x$ alleles by the suppressors. Two lines of evidence suggest that this is unlikely. First, the suppression of the lethal $A x$ combinations does not seem to be allele-specific. We find that all lethal $A x$ combinations are rescued by these suppressors. Second, $\mathrm{Dl}$ and mam mutants have diverse effects on the phenotypes of individual $A x$ mutations. $D l$ alleles generally suppress the Ax phenotype, whereas mam alleles, although they suppress the negative complementation lethality, do not have any effect on the wing phenotype of an individual $A x$ mutation. Moreover, the $E(s p l)$ deletions, which suppress the phenotype of $A x$ alleles, have no effect on the negative complementation. Because these data argue against the simple inactivation of an $A x$ allele by the suppressors, a reasonable working hypothesis is that the rescue reflects an interference with the interaction between the $A x$ mutations per se. For instance, the interaction between two negatively interacting molecules is either eliminated or rendered less severe, resulting in a viable phenotype.

Fuller and her collaborators have carried out elegant screens in Drosophila, demonstrating that mutations in $\beta$-tubulin fail to complement mutations in $\alpha$-tubulin, two molecules known to form heterodimers (Hays et al. 1989). Thus, they showed directly that mutations in separate genes coding for interacting molecules can fail to complement each other. In an analogous fashion, the findings we report here raise the possibility of specific associations between $N$ and $D l$ as well as $N$ and mam. In this respect, it is particularly interesting that $D l$ was shown to code for a transmembrane protein with EGF homology (Vässin et al. 1987; Kopczynski et al. 1988). If we assume that $N$ protein function depends on both homotypic interactions and interactions between $N$ and $D l$ or mam molecules, a conceivable way that the reduction of $\mathrm{Dl}$ or mam dosage could influence two negatively interacting $N$ molecules is by directly influencing the binding constant between those $N$ molecules. This could be attained, for example by reducing the concentration of one of the members in a particular molecular assembly.

The $D l$ and mam mutations recovered from the screen have not yet been fully examined. It should be mentioned that because we have used ENU as the mutagen, we expect many, if not all, of the suppressors isolated in this screen to involve point mutations (Batzer et al. 1988). Preliminary examination of some of the $D l$ alleles suggests the existence of hypomorphic mutations in this group (M.A.T. Muskavitch, pers. comm.). Although these mutations have apparently not lost all function, they behave as deletions in terms of rescuing negative complementation. It will be particularly interesting to analyze suppressors that are hypomorphic $D I$ and mam point mutations because such lesions may point to domains important for protein-protein interactions.

As expected, most of the suppressors that we isolated mapped to the $N$ locus. We are currently analyzing these lethal $N$ alleles to establish the nature of the molecular lesion and the exact phenotypes associated with them. A nonsense mutation in $N$ that results in a completely nonfunctional product would be identified in our screen as a suppressor, because the $A x$ alleles that we examined are viable over a $N$ deficiency. Suppressors involving second-site missense mutations in the $N$ protein will be of special value for studying structure/function relationships in the $N$ locus. An initial phenotypic characterization of these new $N$ alleles suggests that they can be classified into different mutant categories according to their embryonic and adult phenotypes. Correlating such classification with specific molecular lesions can be of obvious importance.

Previous work has revealed the existence of interactions between $N$ and $E(s p l)$ as well as $E(s p l)$ and $D l$ (Welshons 1956; Vässin et al. 1985; Shepard et al. 1989). de la Concha et al. (1988) compared the severity of discrete neurogenic mutations in the presence and absence of large chromosomal duplications carrying an extra copy of a different neurogenic locus. They interpret the results of their studies to suggest directed functional relationships between all of the neurogenic loci except bib. In the present studies, both the results of the genetic screen and the phenotypic examination of specific double mutant combinations suggest functional relationships between $N, D I, m a m$, and $E(s p l)$. According to the criteria used in the present analysis, which are different and less constrained than those used in previous studies, we detect a complex, allele-specific pattern of interactions between $N$ and the three neurogenic loci $D I$, mam, and $E$ (spl) but do not detect interactions between any $N$ allele and either bib or neu.

In addition to the interaction between the $A x$ alleles and the lethal point mutations of the $E(s p l)$ region, which affect the transducin homologous transcript, a dramatic synergistic effect was observed between the point mutation $E(s p l)^{E 73}$ and two missense mutations, $n d$ and $n d^{2}$, which were shown to affect the intracellular portion of the $N$ protein. This observation invites the speculation that specific molecular interactions exist between the intracellular domain of the $N$ protein and the transducin homologous gene product of the $E(s p l)$ region. Similarly, nd and $n d^{2}$ interact with mam, whose molecular nature is not yet known. When considering the specificity of such interactions, it should be kept in mind that $n d$ was recently reported to interact with both scabrous and vestigial (Rabinow and Birchler 1990). The phenotypic result of these interactions is reminiscent of that seen between $n d$ and $E(s p l)$ or mam.

An important consequence of the clear synergistic phenotypes seen with the $n d$ alleles is that they will allow us to expand the basic approach that we have followed here in searching for interacting elements. Namely, one can now search for interactions between three, rather than two, genetic loci. That is, using a 
double mutant combination of an interacting genetic pair, one can attempt to identify additional elements that alter the double mutant phenotype. We believe that this is an important extension of the present work, which will be potentially very informative (D. Hartley, in prep.).

In summary, the results of the screen and the subsequent analyses revealed specific relationships between $N$ and $D l, m a m$, and $E(s p l)$, which suggests that they may be functionally related at the protein level. In fact, our preliminary biochemical analyses indicate that the $N$ and $D l$ proteins may be directly associated with each other (R. Fehon, J. Kooh, I. Rebay, C.L. Regan, T. Xu, M.A.T. Muskavitch, and S. Artavanis-Tsakonas, unpubl.). Given the results of the present analysis, this is not surprising nor would it be surprising to find that in addition to $D l$, the $N$ protein may interact with the products of mam as well as with the transducin-like product of $E(s p l)$.

\section{Materials and methods}

\section{Mutagenesis}

A total of $2,100 A x^{E 2} s n^{3}$ virgins were mated to $900 \mathrm{ENU}$ treated (Sigma) y $w A x^{9 B 2}$ males using a modification of a protocol described by Lipshitz (1975). All of the parental males were removed from the bottles after having mated for 4 days. Thirty-six fertile heterozygous females were collected from among $12,946 A x^{E 2} s n^{3}$ sibling males. Individual lines were established from each female. Twenty-three suppressors were mapped to the $\mathrm{X}$ chromosome. Twenty-one of them were recessive lethals and because their lethalities were rescuable by a transformant that contains $N$ DNA sequences (Ramos et al. 1989), we concluded that they are $N$ alleles. Four recessive lethal suppressors that have been mapped to the second chromosome behaved as mam alleles because they failed to complement each other and also failed to complement mam ${ }^{I L 115}$. They were named $\mathrm{mam}^{88-4}, \mathrm{mam}^{88-10}, \mathrm{mam}^{88-31}$, and $\mathrm{mam}^{88-44}$. The nine remaining suppressors represented one recessive lethal complementation group on the third chromosome, and all of them showed a dominant DI-like wing phenotype. The ability to suppress negative complementation, as well as the dominant wing phenotype of one of the third chromosomal suppressors, was mapped between stripe and ebonys using the rucuca chromosome. Given that all nine suppressors failed to complement $D I$ mutations $\left[D I^{9 P 39}\right.$ and $\left.D f(3 R) D 1^{B X 6}\right]$, we concluded that they were $D l$ alleles. They were consequently named $D 1^{88-16}, D 1^{88-20}, D 1^{88-28}, D 1^{88-32}, D 1^{88-34}, D 1^{88-35}, D 1^{88-46}$, $D 1^{88-47}$, and $D 1^{88-50}$. An additional $D 1$ allele $\left(D 1^{88-49}\right)$ was recovered as a phenotypically singed non- $A x$ Fl male from the genetic screen.

\section{Strains and crosses}

Stocks were maintained and crosses were performed on a standard cornmeal $/$ molasses/yeast $/$ agar medium containing $0.2 \%$ propionic acid, or Tegosept, as mold inhibitors. All cultures were maintained at $25^{\circ} \mathrm{C}$, unless specific temperatures were mentioned. Genetic markers and strains not specifically mentioned are found in Lindsley and Grell (1968) or in Lindsley and Zimm (1985). $A x^{9 B 2}$ was previously reported as female-sterile. We have found that the sterile phenotype is due to another mutation on the same chromosome. Homozygous $A x^{9 B 2}$ are fully fertile after recombining off the female sterile mutation.

The strains and crosses for the data in Figure 2 are given below.

The crosses involving $D l$ mutations were:

$A x^{E 2} s^{3} ; D f(3 R) D 1^{B X 6}, D 1^{9 P 39}$ or $D 1^{88 ' s / T M 1}$ virgins $\times y w$ $A x^{9 B 2} / Y$ males.

y $w A x^{9 B 2} / F M 7 C$ virgins $\times A x^{E 2} s n^{3 / Y} ; D f(3 R) D l^{B X 6}$ or $D 1^{9 P 39} / T M 1$ males.

$A x^{71 d} s n^{3}$ virgins $\times$ y $w A x^{9 B 2} / Y ; D l^{88-20} / T M 1$ males.

y $w A x^{9 B 2} / F M 7 C$ virgins $\times$ y $w A x^{16172} \Delta / Y ; D 1^{88-20} /+$ males.

$A x^{75 c 24} / y^{2} M-5$ virgins $\times A x^{E 2} s n^{3 / Y} ; D 1^{88-20} / T M 1$ males.

y w $^{a} \mathrm{fa}^{8} A \mathrm{x}^{59 d} / F M 6$ virgins $\times A x^{E 2} s n^{3 / Y} ; D 1^{88-49} / T M 6 B$ or $A \mathrm{X}^{E 2} s n^{3 / Y} ; D l^{9 P 39}, D l^{B X 6}$, or $D 1^{88^{\prime}} / T M 1$ males.

y $w A x^{16172}$ virgins $\times$ y $w A x^{16172 / Y} ; D 1^{88-20} /+$ males.

The crosses involving mam mutations were:

$A x^{E 2} s n^{3} ;$ cn bw sp $\operatorname{mam}^{I L 115}$ or $\operatorname{mam}^{88 ' s / S M 1}$ virgins $\times$ y $w$ $A x^{9 B 2 / Y}$ males.

y $w A x^{9 B 2} / F M 7 C$ virgins $\times A x^{E 2} s n^{3 / Y}$; cn bw sp mam ${ }^{I L 115 /}$ SM1 males.

y $w A x^{9 B 2} / F M 6 ; c n$ bw sp mam ${ }^{I L 115 / S M 1}$ virgins $\times$ y $w$ $A x^{16172} / Y$ or $A x^{71 d} s n^{3} / Y$ males.

The crosses involving $E(s p l)$ deficiencies were:

$A x^{E 2} s n^{3} ; E(s p l)^{R 1}$ or $1(\text { gro })^{X 1 / T M 1}$ virgins $\times$ y $w A x^{9 B 2} / Y$ males.

y $w A x^{9 B 2} / F M 7 C$ virgins $\times A x^{E 2} s n^{3 / Y} ; E(s p l)^{R 1}$ or $1(\text { gro })^{X 1} /$ TM1 males.

y $w A x^{9 B 2} / F M 6 ; 1(g r o)^{X 1} / T M 2$ virgins $\times A x^{71 d} s n^{3 / Y}$ or y $w$ $A x^{16172 / Y}$ males.

The crosses involving $E(s p l)$ point mutations were:

$A x^{E 2} s n^{3} ; e^{4} E(s p l)^{E 73} t x / T M 1$ virgins $\times$ y w $A x^{9 B 2} / Y$ males.

y $w A x^{9 B 2} / F M 7 C$ virgins $\times A x^{E 2} s n^{3 / Y} ; e^{4} E(s p l)^{E 73} t x / T M 1$ males.

y $w A x^{9 B 2} / F M 6$ or $A x^{E 2} s n^{3}$ virgins $\times e^{4} E(s p l)^{E 48}, E(s p l)^{E 73}$, $E(s p l)^{E 107}$ tx/TM6B males.

y $A \mathrm{x}^{9 B 2} s n^{3}$ virgins $\times e^{4} E(s p l)^{E 73} t \mathrm{x} / T M 6 B$ males.

The crosses involving $E(s p l)^{D}$ were:

$A x^{E 2} s^{3} ; E(s p l)^{D / T M 1}$ virgins $\times$ y $w A x^{9 B 2} / Y$ males. y $w A x^{9 B 2} / F M 7 C$ virgins $\times A x^{E 2} s n^{3 / Y ; E}(s p 1)^{D / T M 1}$ males.

The crosses involving the transformed line that contains DNA sequences from the transducin homologous transcript of the $E(s p I)$ locus were:

$A x^{E 2} \mathrm{sn}^{3} ; P\left[\mathrm{ry}^{+} ; E 8\right][47 B] / S M 1$ virgins $\times$ y ${ }_{w} A x^{9 B 2} / Y ; P\left[r y^{+} ;\right.$ $E 8][47 B] / S M 1$ males.

y $A x^{9 B 2} s n^{3}$ virgins $\times A x^{E 2} s n^{3} / Y ; P\left[r y^{+} ; E 8\right][47 B] / S M 1$ males.

The crosses involving bib mutations were:

$A x^{E 2} s^{3}$; cn $b w$ sp $b i b^{I D 05 / S M 1}$ virgins $\times$ y $w A x^{9 B 2} / Y$ males. y $w A x^{9 B 2} / F M 7 C$ virgins $\times A x^{E 2} s n^{3 / Y}$; cn bw sp bibiD05/SM1 males.

y wAx ${ }^{9 B 2} / F M 6$; cn bw sp bib $b^{1005 / S M 1}$ virgins $\times$ yw $A x^{16172 / Y}$ or $A x^{71 d} s n^{3} / Y$ males.

The crosses involving neu mutations were:

$A x^{E 2} s n^{3} ; n e u^{I F 65 / T M 1}$ virgins $\times$ y $w A x^{982} / Y$ males. y w $A x^{9 B 2} / F M 7 C$ virgins $\times A x^{E 2} s n^{3} / Y$; $n e u^{I F 65 / T M 1}$ males. $y$ w $A x^{9 B 2} / F M 6$; neu ${ }^{I F 65} / T M 2$ virgins $\times$ y w $A x^{16172 / Y}$ or $A x^{71 d}$ $s n^{3} / Y$ males.

The strains and crosses for the data in Figure 3 are as follows: $f a^{g} r b, f a^{n o} r b, w s p l$ or $w^{a} n d$ virgins mated to $D f(3 R) D l^{B X 6}$ / 
TM3Ser, cn bw sp mam ${ }^{I 115 / C y O}$, 1(gro $)^{X 1} / T M 6 B, e^{4} E(s p 1)^{E 73}$ tx/TM6B, E(spl)D, cn bw sp bibiDos/CyO or neu $u^{I I I a} / T M 3$, Ser males.

$n d^{2}$ virgins mated to $D 1^{9 P 39} / T M 1$, cn bw sp mam $^{I L 115} / C y O$, 1(gro $)^{X 1 / T M 6 B, ~} e^{4} E(s p l)^{E 73}$ tX/TM6B, E(spl)D , cn bw sp bib ${ }^{I D 05 /}$ CyO or $n e u^{I F 65 / T M 3}$, Ser males.

$f a^{g} r b, w s p l$, or $n d^{2}$ virgins mated to $E(s p l)^{B X 22} / T M 6 B$ males. $w$ spl virgins mated to mam $^{88-4} / S M 1$ or mam ${ }^{88-10} / S M 1$ males. y $w^{a} N^{5419} / F M 6$ virgins mated to $D l^{9 P 39} / T M 1$, cn bw $s p$ mam $^{1 L 115} / \mathrm{CyO}, 1(\mathrm{gro})^{X 1} / \mathrm{TM} 6 \mathrm{~B}, e^{4} E(\mathrm{spl})^{E 73}$ tx/TM6B, E(spl), $\mathrm{cn}$ bw sp bib ${ }^{O D 05} / \mathrm{CyO}$ or neu IF65/TM3, Ser males.

\section{DNA isolation and sequencing}

Genomic libraries of SacI-digested or SacI- and XhoI-digested DNA from $w^{a} n d$ or $n d^{2}$ homozygous adult flies were cloned in the SacI or SacI and XhoI sites of $\lambda$ ZAP /Stratagene Cloning Systems) and screened with ${ }^{32}$-labeled $N$ probes (Maniatis et al. 1982) to isolate mutant DNA. Bluescript SK plasmids were released from $\lambda$ ZAP by superinfecting with M13 helper phage.

Double-stranded DNAs from these clones were primed with synthetic primers derived from wild-type sequences or the M13 universal primer and sequenced by the dideoxynucleotide chain-termination procedure (Sanger et al. 1977; Toneguzzo et al. 1988) using Sequenase (U.S. Biochemical). In most cases, only the sense strand was sequenced.

\section{Acknowledgments}

We acknowledge the help of Kristi Wharton in the initial stages of the nd mutant analysis. We are grateful to all of our colleagues in the Artavanis laboratory, as well as the Kankel laboratory, for valuable suggestions, and we acknowledge the expert technical assistance of Ms. Ruth Schlesinger-Bryant and Wan Yu. T.X. was supported by a Yale University doctoral fellowship, I.R. by a Howard Hughes Medical Institute doctoral fellowship, and T.N.S. and R.J.F. were supported by National Institutes of Health (NIH) postdoctoral fellowships. This research was supported by NIH grants GM-29093 and NS-26084 and National Science Foundation grant DCB-8713241, awarded to S.A.-T.

\section{References}

Alton, A.K., K. Fechtel, C.C. Kopczynski, S.B. Shepard, P.J. Kooh, and M.A.T. Muskavitch. 1989. Molecular genetics of Delta, a locus required for ectodermal differentiation in Drosophila. Dev. Genet. 10: 261-272.

Artavanis-Tsakonas, S. 1988. The molecular biology of the Notch locus and the fine tuning of differentiation in Drosophila. Trends Genet. 4: 95-100.

Batzer, M.A., B. Tedeschi, N.G. Fossett, A. Tucker, G. Kirloy, P. Arbour, and W.R. Lee. 1988. Spectra of molecular changes induced in DNA of Drosophila spermatozoa by 1-ethyl-1-nitrosourea and X-rays. Mut. Res. 199: 255-268.

Breeden, L. and K. Nasmyth. 1987. Similarity between cell cycle genes of budding yeast and fission yeast and the Notch gene of Drosophila. Nature 329: 651-654.

Cagan R.L. and D.F. Ready. 1989. Notch is required for successive cell decisions in the developing Drosophila retina. Genes Dev. 3: 1099-1112. de la Concha, A., U. Dietrich, D. Weigel, and J.A. Campos-Ortega. 1988. Functional interactions of neurogenic genes of Drosophila melanogaster. Genetics 118: 499-508.

Doe, C.Q. and C.S. Goodman. 1985. Early events in insect neurogenesis II. The role of cell interactions and cell lineage in the determination of neuronal precursor cells. Dev. Biol. 111: 206-219.

Foster, G.G. 1975. Negative complementation at the Notch locus of Drosophila melanogaster. Genetics 81: 99-120.

Hartenstein, V. and J.A. Campos-Ortega. 1984. Early neurogenesis in wild-type Drosophila melanogaster. Wilhelm Roux's Arch. Dev. Biol. 193: 308-325.

Hartley, D.A., T. Xu, and S. Artavanis-Tsakonas. 1987. The embryonic expression of the Notch locus of Drosophila melanogaster and the implications of point mutations in the extracellular EGF-like domain of the predicted protein. $E M B O$ I. 6: 3407-3417.

Hartley, D.A., A. Preiss, and S. Artavanis-Tsakonas. 1988. A deduced gene product from the Drosophila neurogenic locus, Enhancer of split, shows homology to mammalian Gprotein beta subunit. Cell 55: 785-795.

Hays, T.S., R. Deuring, B. Robertson, M. Prout, and M. Fuller. 1989. Interacting proteins identified by genetic interactions: A missense mutation in $\beta$-tubulin gene of Drosophila melanogaster. Mol. Cell. Biol. 9: 875-884.

Held, L.I. Jr. and P.J. Bryant. 1984. Cell interactions controlling the formation of bristle patterns in Drosophila. Pattern formation (ed. G.M. Malacinski and S.V. Bryant.) pp. 291-322. Macmillan, New York.

Johansen, K., R.G. Fehon, and S. Artavanis-Tsakonas. 1989. The Notch gene product is a glycoprotein expressed on the cell surface of both epidermal and neuronal precursor cells during Drosophila development. I. Cell Biol. 109: 24272440.

Jürgens, G., E. Wieschaus, C. Nüsslein-Volhard, and H. Kluding. 1984. Mutations affecting the pattern of larval cuticle in Drosophila melanogaster. II. Zygotic loci on the third chromosome. Wilheim Roux's Arch. Dev. Biol. 193: 283-295.

Kelley, M.R., S. Kidd, W.A. Deutsch, and M.W. Young. 1987. Mutations altering the structure of epidermal growth factorlike coding sequences at the Drosophila Notch locus. Cell 51: $539-548$.

Kidd, S. and M.W. Young. 1986. Transposon-dependent mutant phenotypes at the Notch locus of Drosophila. Nature 323: $89-91$.

Kidd, S., M.R. Kelley, and M.W. Young. 1986. Sequence of the Notch locus of Drosophila melanogaster: Relationship of the encoded protein to mammalian clotting and growth factors. Mol. Cell. Biol. 6: 3094-3108.

Kidd, S., M.K. Baylies, G.P. Gasic, and M.W. Young. 1989. Structure and distribution of the Notch protein in developing Drosophila. Genes Dev. 3: 1113-1129.

Klaembt, C., E. Knust, K. Tieze, and J.A. Campos-Ortega. 1989. Closely related transcripts encoded by the neurogenic gene complex Enhancer of split of Drosophila melanogaster. EMBO I. 8: 203-210.

Knust, E., K. Tietze, and J.A. Campos-Ortega. 1987. Molecular analysis of the neurogenic locus Enhancer of split of Drosophila melanogaster. EMBO I. 6: 4113-4123.

Kopczynski, C.C., A.K. Alton, K. Fechtel, P.J. Kooh, and M.A.T. Muskavitch. 1988. Delta, a Drosophila neurogenic gene, is transcriptionally complex and encodes a protein related to blood coagulation factors and epidermal growth factor of vertebrates. Genes Dev. 2: 1723-1735.

Lehmann, R., F. Jimenez, U. Dietrich, and J.A. Campos-Ortega. 
1983. On the phenotype and development of mutants of early neurogenesis in Drosophila melanogaster. Wilheim Roux's Arch. Dev. Biol. 192: 62-74.

Lindsley, D.L. and E.H. Grell. 1968. Genetic variations of Drosophila melanogaster. Carnegie Inst. Washington Publ. No. 627.

Lindsley, D. and G. Zimm. 1985. The genome of Drosophila melanogaster. Dros. Inf. Serv. No. 62.

Lipshitz, H. 1975. Specificity of gene action during the development of the nervous system of Drosophila melanogaster. Ph.D. thesis, Yale University.

Maniatis, T., E.F. Fritsch, and J. Sambrook. 1982. Molecular cloning: A laboratory manual. Cold Spring Harbor Laboratory Press, Cold Spring Harbor, New York.

Markopoulou, K. and S. Artavanis-Tsakonas. 1989. The expression of the neurogenic locus Notch during the imaginal development of Drosophila melanogaster and its relationship to mitotic activity. J. Neurogenet. 6(1): 11-26.

Markopoulou, K., W.J. Welshons, and S. Artavanis-Tsakonas. 1989. Morphological and genetic characterization of the facets: A group of intronic mutations in the Notch locus. Genetics 122: 417-428.

Nüsslein-Volhard, C. and E. Wieschaus. 1980. Mutations affecting segment number and polarity in Drosophila. Nature 287: 795-801.

Nüsslein-Volhard, C., E. Wieschaus, and H. Kluding. 1984. Mutations affecting the pattern of larval cuticle in Drosophila melanogaster. I. Zygotic loci on the second chromosome. Wilheim Roux's Arch. Dev. Biol. 193: 267-282.

Portin, P. 1975. Allelic negative complementation at the Abruptex locus of Drosophila melanogaster. Genetics 81: $121-133$.

Poulson, D.F. 1937. Chromosomal deficiencies and embryonic development of Drosophila melanogaster. Proc. Natl. Acad. Sci. 23: 133-137.

-1950. Histogenesis, organogenesis and differentiation in the embryo of Drosophila melanogaster. In Biology of Drosophila (ed. M. Demerec) pp. 168-274. Wiley, New York.

Preiss, A., D.A. Hartley, and S. Artavanis-Tsakonas. 1988. The molecular genetics of Enhancer of split, a gene required for embryonic neural development in Drosophila. EMBO J. 7: 3917-3927.

Rabinow, L. and J.A. Birchler. 1990. Interactions of vestigial and scabrous with the Notch locus of Drosophila melanogaster. Genetics (in press).

Ramos, R.G.P., B. Grimwade, K.A. Wharton, T.N. Scottgale, and S. Artavanis-Tsakonas, 1989. Physical and functional definition of the Drosophila Notch locus by P- element transformation. Genetics 123: 337-348.

Sanger, F., S. Nicklen, and A.R. Coulson. 1977. DNA sequencing with chain-terminating inhibitors. Proc. Natl. Acad. Sci. 74: 5463-5467.

Shellenbarger, D.L. and J.D. Mohler. 1975. Temperature-sensitive mutations of the Notch locus in Drosophila melanogaster. Genetics 81: 143-162.

1978. Temperature-sensitive periods and autonomy of pleiotropic effects of $1(1) N^{t s}$, a conditional Notch lethal in Drosophila. Dev. Biol. 62: 432-446.

Shepard, S.B., S.A. Broverman, and M.A.T. Muskavitch. 1989. A tripartite interaction among alleles of Notch, Delta and Enhancer of split during imaginal development of Drosophila melanogaster. Genetics 122: 429-438.

Technau, G.M. and J.A. Campos-Ortega. 1986. Lineage analysis of transplanted individual cells in embryos of Drosophila melanogaster. II. Commitment and proliferative abilities of epidermal and neural cell precursors. Wilheim Roux's Arch.
Dev. Biol. 195: 445-454.

Tomlinson, A. and D.F. Ready. 1987. Neuronal differentiation in the Drosophila ommatidium. Dev. Biol. 120: 366-376.

Toneguzzo, F., S. Glynn, E. Levi, S. Mjolsness, and A. Hayday. 1988. Use of a chemically modified T7 DNA polymerase for manual and automated sequencing of supercoiled DNA. BioTechniques 6: 460-469.

Vässin, H., J. Vielmetter, and J.A. Campos-Ortega. 1985. Genetic interactions in early neurogenesis of Drosophila melanogaster. I. of Neurogenesis 2: 291-308.

Vässin, H., K.A. Bremer, E. Knust, and J.A. Campos-Ortega. 1987. The neurogenic gene Delta of Drosophila melanogaster is expressed in the neurogenic territories and encodes a putative transmembrane protein with EGF-like repeats. $E M B O$ \%. 6: $3431-3440$.

Welshons, W.J. 1956. Dosage experiments with split mutants in the presence of an enhancer of split. Dros. Inf. Serv. 30: $157-158$.

-1965. Analysis of a gene in Drosophila. Science 150: 1122-1129.

1971. Genetic basis for two types of recessive lethality at the Notch locus in Drosophila. Genetics 68: 259-268.

Wharton, K.A. 1986. A fine structural analysis of the Notch locus in Drosophila melanogaster. Ph.D. thesis, Yale University.

Wharton, K.A., K.M. Johansen, T. Xu, and S. Artavanis-Tsakonas. 1985a. Nucleotide sequence from the neurogenic locus Notch implies a gene product that shares homology with proteins containing EGF-like repeats. Cell 43: 567581 .

Wharton, K.A., B. Yedvobnick, V.G. Finnerty, and S. ArtavanisTsakonas. 1985b. opa: A novel family of transcribed repeats shared by the Notch locus and other developmentally regulated loci in Drosophila melanogaster. Cell 40: 55-62.

Wieschaus, E., C. Nüsslein-Volhard, and G. Jürgens. 1984. Mutations affecting the pattern of larval cuticle in Drosophila melanogaster. II. Zygotic loci on the X-chromosome and fourth chromosome. Wilheim Roux's Arch. Dev. Biol. 193: $296-307$ 


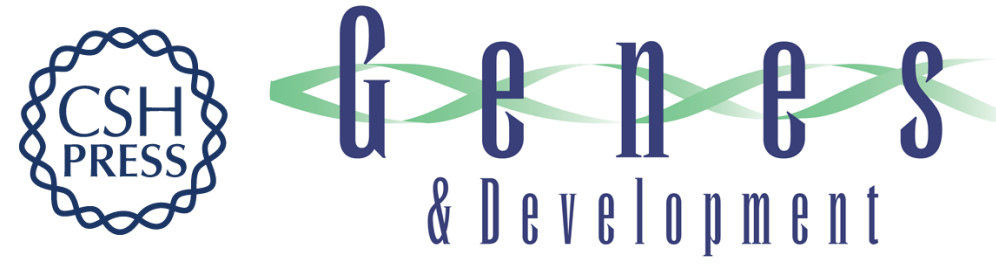

\section{The Notch locus and the genetic circuitry involved in early Drosophila neurogenesis.}

T Xu, I Rebay, R J Fleming, et al.

Genes Dev. 1990, 4:

Access the most recent version at doi:10.1101/gad.4.3.464

References This article cites 45 articles, 17 of which can be accessed free at: http://genesdev.cshlp.org/content/4/3/464.full.html\#ref-list-1

License

Email Alerting

Service

Receive free email alerts when new articles cite this article - sign up in the box at the top right corner of the article or click here.

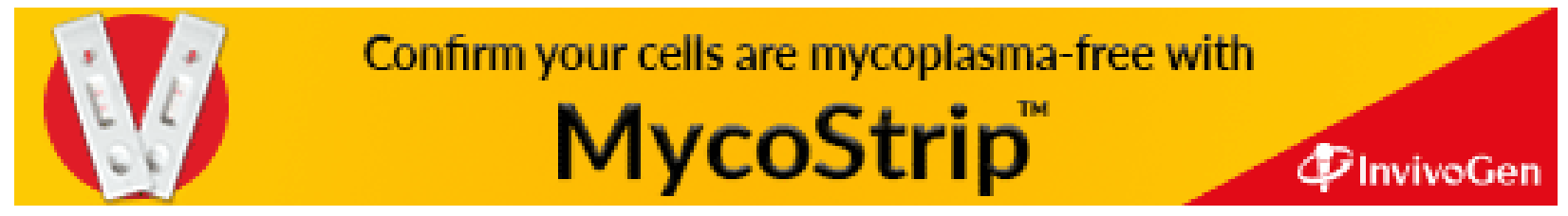

